\title{
PERCEPCIÓN DE LAS PERSONAS TRANSEXUALES SOBRE SU INTEGRACIÓN SOCIAL
}

\author{
Alejandro Domingo Hernández Melián \\ Universidad de La Laguna \\ alejandro.hdez.melian@gmail.com
}

\section{RESUMEN}

Las personas transexuales han formado y forman un grupo de especial vulnerabilidad en las sociedades, observándose la necesidad de realizar estudios exhaustivos y detallados de esta realidad. La presente investigación tiene como objetivo conocer las barreras y facilitadores presentes durante la integración social de las personas transexuales. Para ello se utiliza un método mixto realizando entrevistas semiestructuradas y cuestionarios para la recogida de datos. Los participantes son personas transexuales que residen en la Comunidad Autónoma de Canarias. Los resultados muestran cómo la atención sanitaria especializada y el entorno familiar actúan como ejes centrales positivos, mientras que la carencia de información y formación por parte de las instituciones y profesionales generan impedimentos durante el proceso.

Palabras Clave: personas transexuales, integración social, barreras, facilitadores.

\section{PERCEPTION OF TRANSSEXUAL PEOPLE ABOUT \\ THEIR SOCIAL INTEGRATION}

\section{Abstract}

Transsexual people have formed and form a group of special vulnerability in societies, that is why there is a need of doing exhaustive detailed studies of this reality. This research aims to know the barriers and facilitators during the social integration of transsexual people. In order to achieve this, a mixed method is used to do semistructured interviews and online surveys to collect data. Participants are transsexual people living in the region of the Canary Islands The results show how both the specialized health attention and the family act as central positive axes, while the formation of information and the training by the institutions and professionals generate impediments during the process. The social work plays an important role in the attention of transsexual people by the great social component of the needs that they present.

KEYWORDs: transsexual people, social integration, barriers, facilitators. 


\section{INTRODUCCIÓN Y JUSTIFICACIÓN}

Las personas transexuales son un grupo bastante discriminado en nuestro país (Armaza y Atienza, 2014; Centro de Investigaciones Sociológicas, 2013; Esteva et al., 2012; European Commission, 2015; Fernández, 2010; Herrero y Díaz, 2009; Martín, 2006), sufriendo multitud de dificultades a lo largo de su vida. La visibilización actual del colectivo en la sociedad ha llegado para quedarse, pero esta no se traduce precisamente en sensibilización social, siendo, la transexualidad, estigmatizada y patologizada en diversos contextos. $\mathrm{Y}$ es en este punto en el que convergen multitud de dificultades y problemáticas. Por tanto, conocer cuál es la visión que se tiene desde el propio colectivo acerca de su integración social facilitaría detectar los puntos clave sobre los que intervenir, identificando las barreras y los facilitadores existentes en diferentes ámbitos, utilizando los resultados de esta investigación para orientar la práctica profesional y la implementación de programas de inclusión con esta población, gestionando y utilizando los recursos existentes de una manera más óptima y acorde a la realidad presentada.

El objeto de estudio de esta investigación es el análisis de las percepciones de las personas transexuales durante su integración social, concibiéndola como la trayectoria vital de los sujetos hacia su autonomía y plena participación como adultos en la sociedad de la que forman parte, que abarca contextos tales como las problemáticas sanitarias, el mercado laboral y la inserción en el mismo, las relaciones familiares, sociales y con las instituciones. Cada uno de estos aspectos en su individualidad, y todos ellos en su conjunto, dibujan un marco que no solo atañe a lo económico, sino a lo personal y lo emocional de las personas.

En primer lugar, es importante aclarar la diferencia entre sexo y género, haciendo referencia el primero a los caracteres sexuales externos de las personas, es decir, genitales o rasgos físicos (Herrero y Díaz, 2009), y el segundo a "las prácticas, símbolos, espacios, normas, valores y atributos que la sociedad elabora y construye para y a partir de la diferencia entre hombre y mujer» (Rubio, 2009: 367).

Asimismo, la identidad sexual es el sentimiento íntimo de una persona de ser hombre o mujer. En otras palabras, el sexo sentido de cada individuo (Aventín, 2015).

De igual modo, la orientación sexual es definida como «la atracción y/o deseo duradero hacia otra persona en el plano sexual, romántico, emotivo y/o afectivo. En función del género de la persona hacia la cual se siente atraída se puede catalogar la orientación sexual como homosexual, bisexual o heterosexual» (Alder, 2013: 164).

Y, por último, la transexualidad, la cual es:

La disonancia continua y perseverante entre la identidad sexual y su anatomía, [...] mujer transexual es toda aquella persona que nace mujer (sexo cerebral e identidad sexual) en una anatomía masculina y hombre transexual es toda aquella persona que nace hombre en una anatomía femenina (Álvarez, 2008: 56).

En los últimos años, la generación de bibliografía empírica acerca de la realidad LGTBI, y más concretamente la transexual, ha cobrado especial relevancia, quizás por el creciente material jurídico aprobado en esta materia tanto a 
nivel nacional como en las autonomías. Sin embargo, continúan siendo escasas las referencias en aspectos más concretos. Pese a ello, se expone brevemente la situación actual del colectivo.

Comenzando por el contexto jurídico internacional, se establece como punto de inflexión la Resolución del Parlamento Europeo de 1989, sobre la discriminación de los transexuales, que considera a esta realidad como un problema de la sociedad, que no sabe hacer frente a un cambio de papeles sexuales culturalmente establecidos. Por esta razón, se solicita a los Estados Miembros de la UE que aprueben disposiciones y legislen para una prohibición explícita de la discriminación hacia estas personas. Partiendo de esta base, y como aseguran Armaza y Atienza (2014), el Tribunal Europeo de Derechos Humanos ha dictado relevantes sentencias a favor de las personas transexuales a las que, a principios del siglo XxI, cuando se carecía de legislación, no les era reconocido el sexo social manifestado por ellas, teniendo como base dichas resoluciones el Convenio Europeo de Derechos Humanos.

A nivel nacional, la Ley 3/2007 de 22 de marzo, reguladora de la rectificación registral de la mención relativa al sexo de las personas, actualmente vigente, permite el cambio del sexo biológico establecido en el Registro Civil, así como el cambio del nombre propio de la persona para que no sea discordante con el sexo mostrado. Los requisitos para dicha rectificación registral que más polémica han suscitado son, por un lado, la obligación de establecer un diagnóstico de disforia de género por parte de un psicólogo clínico o médico; por otro lado, que la persona sea mayor de dieciocho años y, además, que exista un tratamiento durante dos años por parte de la persona para adaptar las características físicas con las del sexo deseado.

Por su parte, Canarias es una de las once comunidades que disponen de legislación específica en transexualidad, más concretamente la Ley 8/2014, de 28 de octubre, de no discriminación por motivos de identidad de género y de reconocimiento de los derechos de las personas transexuales, teniendo por objeto el asegurar que las personas transexuales de las islas reciban una atención integral y adecuada por parte de las administraciones públicas, entre la que encuentra la atención laboral en cuanto a la no discriminación en el trabajo, medidas de discriminación positiva y reconocimiento de las dificultades para acceder a un empleo por parte de las personas transexuales, entre otras.

En cuanto al ámbito sanitario, uno de los últimos estudios realizados es el de Esteva et al. (2012), que recoge cómo nueve de diecisiete autonomías poseen Unidades de Atención a la Transexualidad. Se retrata que en nuestro país «los pacientes transexuales han estado hasta fechas recientes fuera del sistema sanitario público. Algunos conseguían ser operados por cirujanos privados, pero la organización y atención de estos pacientes carecía de rigor disciplinario» (204). Los resultados muestran cómo un $55 \%$ de las comunidades autónomas poseen un protocolo de atención a los problemas derivados de la identidad de género, siendo solo cuatro de ellas las que ofrecen cirugía genitoplástica. Desde el año 2008, en Canarias se contempla el diagnóstico y tratamiento hormonal, pero en cuanto a intervenciones se refiere, solo se ofrece histerooforectomías en los casos que se denominan en el estudio como «transexualidad mujer-hombre». La conclusión del estudio es que, 
en la actualidad, el personal sanitario español dispone de muy poca información y/o formación sobre la transexualidad, salvo aquellos implicados en las unidades especializadas existentes.

Las relaciones familiares son otro contexto fundamental. Vidal (2006), desde la experiencia registrada en la Unidad Especializada del Hospital Clinic de Barcelona, afirma que "el paciente que se encuentra completamente solo en el proceso de reasignación de cambio de sexo, sin apoyo social, aumenta su sufrimiento haciéndolo más vulnerable a padecer trastornos psiquiátricos ansioso-depresivos» (368). Fernández (2010), investiga la realidad de 120 personas transexuales del territorio español. Sus resultados reflejan cómo un 36\% afirma que no habla con nadie del entorno familiar de su situación, siendo el segundo ítem en importancia. Se pone de relieve, así, la imposibilidad de numerosas personas transexuales de tratar asuntos relativos a la identidad sexual con la familia. Por último, Martín (2006) considera que cuando la familia es conocedora de que su hijo/a es transexual, una parte importante reacciona con rechazo, aunque posteriormente aceptan la realidad y le apoyan durante el proceso. Además, añade que actualmente "existe un cambio social, educacional y laboral en estas nuevas generaciones, una mayor posibilidad de realizar estudios medios y universitarios y por lo tanto una mayor posibilidad de una carrera profesional y una mayor integración social» (45).

En lo relativo al contexto laboral y social, hay diversas fuentes aportan datos relevantes. A nivel internacional, el Eurobarómetro (2015) recoge cómo un 56\% de los europeos considera totalmente extendida la discriminación en cuestiones de identidad de género mientras que en Espańa lo afirma el 66\% de los participantes en el estudio, según estos resultados, el tipo de discriminación más amplia. A nivel nacional, el Centro de Investigaciones Sociológicas (2013) señala que el 48,7\% de las personas encuestadas considera que es muy o bastante frecuente la discriminación o trato desfavorable por cuestiones de identidad sexual. Esta discriminación se refleja en diferentes ámbitos, como el residencial o comunitario, ya que a un 5,1\% de la población le molestaría mucho o bastante tener como vecino a una persona transexual y un $16 \%$ considera que ser transexual perjudica a la hora de alquilar una vivienda. También, un $16,1 \%$, trata de considerar a la transexualidad como una barrera o impedimento. Asimismo, un 11,6\% afirma que ser transexual perjudica a la hora de acceder a los servicios públicos. En cuanto al ámbito laboral, el 24,9\% de los encuestados establece la transexualidad como factor negativo a la hora de acceder a un puesto de trabajo. Además, un $24 \%$ manifiesta que ser transexual afecta en cuanto al acceso a puestos de responsabilidad.

A la vista de los datos expuestos, que enmarcan el panorama social en el que se encuentra el colectivo en nuestro país, esta investigación pretende indagar sobre las dificultades y los apoyos que se pueden presentar a lo largo del proceso de integración de las personas transexuales en Canarias.

\section{OBJETIVOS}

- Describir el perfil biosociodemográfico de las personas transexuales de Canarias. 
- Identificar las barreras existentes durante el proceso de integración social de las personas transexuales.

- Conocer los facilitadores del proceso de integración social de las personas transexuales.

\section{MÉTODO}

\section{POBLACIÓN Y MUESTRA}

El número total de participantes son 11 personas en las entrevistas y 88 para la realización de los cuestionarios, teniendo los primeros las siguientes características:

\begin{tabular}{|c|c|c|c|c|c|c|c|c|c|}
\hline ¿̊. & 究 & 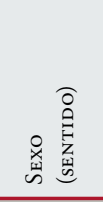 & 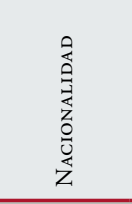 & 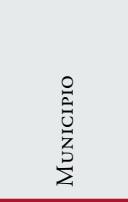 & 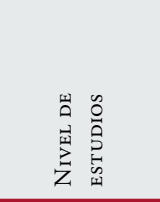 & 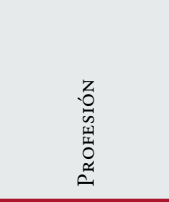 & 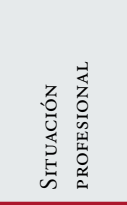 & 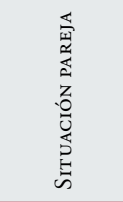 & 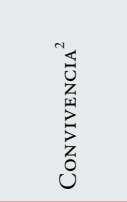 \\
\hline 1 & 23 & Mujer & Española & Candelaria & Secundarios & $\begin{array}{l}\text { Auxiliar de } \\
\text { enfermería }\end{array}$ & Estudiante & Soltera & $\begin{array}{c}\text { F. de } \\
\text { origen }^{3}\end{array}$ \\
\hline 2 & 51 & Mujer & Española & La Laguna & Secundarios & Funcionaria & Empleada & $\begin{array}{c}\text { P. sin } \\
\text { convivir }\end{array}$ & F. de origen \\
\hline 3 & 26 & Hombre & Española & Arona & $\begin{array}{c}\text { Ciclo/Bach } \\
\text { Superior }\end{array}$ & Camarero & En paro & Soltero & F. de origen \\
\hline 4 & 51 & Hombre & Espańola & Santa Cruz & $\begin{array}{c}\text { Ciclo/Bach } \\
\text { Superior }\end{array}$ & Jefe de tráfico & Empleado & Separado & Solo \\
\hline 5 & 30 & $\underset{\text { binario }}{\text { No }}$ & Española & Santa Cruz & $\begin{array}{c}\text { Ciclo/Bach } \\
\text { Superior }\end{array}$ & Educador & Empleado & $\begin{array}{l}\text { P. sin } \\
\text { convivir }\end{array}$ & Solo/a \\
\hline 6 & 18 & $\begin{array}{c}\text { No } \\
\text { binario }\end{array}$ & Española & Santa Cruz & $\begin{array}{c}\text { Ciclo/Bach } \\
\text { Superior }\end{array}$ & Estudiante & Estudiante & $\begin{array}{c}\text { P. sin } \\
\text { convivir }\end{array}$ & F. de origen \\
\hline 7 & 27 & Mujer & Venezolana & Santa Cruz & $\begin{array}{c}\text { Ciclo/Bach } \\
\text { Superior }\end{array}$ & Estilista & En paro & Casada & Pareja \\
\hline 8 & 59 & Mujer & Española & La Orotava & Secundarios & Artesanía & En paro & Divorciada & Sola \\
\hline 9 & 36 & Hombre & Española & Santa Cruz & Secundarios & Camarero & En paro & Soltero & F. de origen \\
\hline 10 & 40 & Hombre & Española & Granadilla & Universitarios & Administrativo & En paro & Soltero & Solo \\
\hline 11 & 25 & Hombre & Española & $\begin{array}{l}\text { Guía de } \\
\text { Isora }\end{array}$ & $\begin{array}{c}\text { Ciclo/Bach } \\
\text { Superior }\end{array}$ & $\begin{array}{l}\text { Diseñador } \\
\text { gráfico }\end{array}$ & Empleado & Soltero & F. de origen \\
\hline
\end{tabular}

Nota: ${ }^{1}$ Número de la entrevista/participante. ${ }^{2}$ Situación convivencial. ${ }^{3}$ Convivencia con la familia de origen. ${ }^{4}$ Con pareja, pero sin convivir. ${ }^{5}$ No se identifica ni como hombre ni como mujer, o con ambos simultáneamente.

Los participantes en la encuesta tienen una edad media de 32,2 años, 55 mujeres y 33 hombres. El 75\% reside en la isla de Tenerife y un $40 \%$ posee estudios universitarios. Además, un $41 \%$ se encuentra soltero/a y un $55,6 \%$ convive con la familia de origen. 
Dado que no existe un censo oficial internacional, nacional o autonómico sobre el número de personas transexuales, para las entrevistas se ha utilizado el muestreo intencional por decisión razonada, seleccionando a los sujetos según los criterios siguientes: persona de 18 o más años, residente en alguna isla del archipiélago canario y que declare su disconformidad entre el sexo asignado al nacer con el sexo sentido.

Para los cuestionarios, se optó por realizar una "bola de nieve» con dos puntos de partida. Por un lado, a partir de la muestra inicialmente acotada para las entrevistas y por otro a través del contacto con informantes clave de diversas organizaciones: Asociación de Personas Transexuales de Tenerife (Aperttura), Asociación de Lesbianas, Gais, Bisexuales, Trans e Intersexuales (Algarabía), Médicos del Mundo, Asociación del Colectivo LGTB+ simpatizantes de Fuerteventura (Altihay) y Colectivo de Lesbianas, Gays, Transexuales y Bisexuales de Canarias (Gamá).

\section{TÉCNICAS E INSTRUMENTOS}

Para la recogida de datos se utilizó una entrevista semiestructurada en profundidad con los apartados siguientes: datos sociodemográficos; manifestación (consciencia y/o expresión del "sexo sentido vs. sexo asignado»); ámbito escolar (experiencia personal): etapa de la adolescencia (relaciones sociales y familiares); asistencia sanitaria (atención desde el sistema público español); mercado laboral (percepción y experiencia); relaciones actuales (sociales y familiares); perspectivas de futuro (expectativas con respecto a la situación actual), junto con un cuestionario, distribuido de manera on-line a todas aquellas personas transexuales de la Comunidad Autónoma de Canarias interesadas en cumplimentarla, que se estructura en dos grandes apartados: datos sociodemográficos y percepción de las personas transexuales de su integración social (ámbitos de vulnerabilidad, discriminación percibida e idoneidad del profesional en trabajo social).

Para el análisis de la información se utilizaron: análisis de contenido en las entrevistas, descripción básica con porcentajes y medias de los datos de los cuestionarios y finalmente se triangularon los datos obtenidos con estas dos técnicas que fueron discutidos con los antecedentes disponibles.

\section{RESULTADOS}

Algunos de los/as entrevistados/as no le contaron a nadie su situación de transexual hasta pasado un tiempo, ya fuera por miedo o por inseguridad. Pero la amplia mayoría lo comentó, por primera vez, en el entorno familiar. En cuanto al proceso, la mayoría ha seguido el protocolo sanitario establecido. Sin embargo, algunas personas afirman que no acudieron a dicho servicio por un conocimiento previo, sino por derivación de otros profesionales.

En cuanto al ámbito escolar, algunas personas definen esta etapa como desagradable, por cuestiones de no afinidad con el sexo asignado. Aunque, como afirman, a esas edades no saben con exactitud qué es lo que les sucede. Las situacio- 
nes que se destacan como especialmente vulnerables en la vida escolar de un menor transexual, y en las que una amplia mayoría de los/as entrevistas/as coinciden, son la referente al nombre, es decir, llamar a un menor por el nombre con el que no se identifica, y el hecho de no dejar que el/la niño/a use el baño que corresponde con su sexo sentido. Además, ańaden que este tipo de situaciones no mejorarán si los profesores, los directores de los centros o las administraciones en general no hacen algo por evitarlo. En general, la percepción que se tiene de las actuaciones de los colegios y de las administraciones públicas canarias para evitar las situaciones de acoso hacia los menores transexuales es muy precaria, afirmando la mayoría que lo que se está haciendo es poco y va a un ritmo muy lento.

En lo referente a la etapa de la adolescencia, se percibe, en general, un empeoramiento de los discursos con respecto a la época escolar cuando se pregunta acerca del recuerdo que se tiene de esta. En las relaciones familiares hay dos grupos. Por un lado, algunas personas declaran que han sido buenas, siendo un poco complicadas en un principio pero que, posteriormente, fueron mejorando. En cambio, otras no tuvieron unas relaciones familiares muy satisfactorias debido a la incomprensión o falta de acercamiento por parte de los padres.

La asistencia sanitaria ha sido muy buena para una amplia mayoría de las personas entrevistadas. Sin embargo, uno de los grandes déficits detectados, y cuestión por la cual se les ha preguntado específicamente, es la gran carencia que se percibe en cuanto a formación del personal sanitario acerca de la transexualidad. Determinadas personas, en cambio, sí creen que poseen formación, pero que aun así les cuesta llevarla a la práctica. Por último, en referencia a los medicamentos hormonales, todas las personas entrevistadas defienden la continuidad de que estén cubiertos por el sistema público, ya que se considera una necesidad y no un lujo o capricho. Sin embargo, algunos creen que este acceso a los medicamentos es deficitario e incluso han llegado a tener problemas para adquirirlos, no por cuestiones de prohibición, sino por falta de abastecimiento en las farmacias, situación que, a la vista de los relatos, puede perjudicar al organismo, al carecer de una hormona que, de manera natural, el cuerpo no produce.

Todas las personas entrevistadas afirman que los transexuales encuentran dificultades para acceder a un puesto de trabajo, lo que parece deberse a distintas causas. Por un lado, se achacan a la personalidad de cada individuo, la cual condicionaría la actitud del empleador/a. Por otra, se asegura que la imagen lo es todo, y que el acceso a los puestos de trabajo es complicado por no estar acorde la corporalidad con el sexo sentido. Además, y continuando con esta idea, varias personas coinciden en distinguir entre las mujeres transexuales y los hombres transexuales cuando se trata del tema laboral, ya que consideran que las mujeres transexuales encuentran mayores dificultades por su aspecto físico, es decir, se percibe de una manera más directa que son transexuales. En relación con la experiencia personal en un puesto de trabajo, muchos han tenido algún problema en un determinado momento, ya sea por dificultad para compaginar el trabajo con las revisiones médicas o por actitudes y comentarios inadecuados por parte de compañeros o superiores. Pero alegan que, al margen de algunos puestos y personas, la realidad vivida es normal. Las personas entrevistadas han identificado diversas actuaciones que se deberían implementar para 
evitar situaciones de discriminación o exclusión en el trabajo, como la facilitación a quienes contratan de información suficiente acerca de la transexualidad para lograr que no se creen estereotipos o, si los hubiera, intentar derribarlos, así como crear cupos laborales a partir de la discriminación positiva de las personas transexuales. También se ve necesaria la imposición de sanciones a aquellas empresas que toleren malas prácticas hacia sus empleados por cuestiones de identidad sexual o de género.

Las relaciones personales y sociales mantenidas en la etapa adulta son notablemente mejores que en la adolescencia. De forma parecida se describen las relaciones familiares como adultas, que son igualmente buenas para la mayoría de las personas. Sin embargo, algunas manifiestan que determinados miembros de su familia no les aceptan, creando conflicto en situaciones concretas.

Todos, sin excepción, afirman rotundamente que el apoyo familiar es fundamental durante el proceso que supone la transexualidad. Pero, a pesar de ello, muchos creen que no es habitual contar con este apoyo, describiendo casos de conocidos o de terceras personas que, por un motivo u otro, no lo han recibido.

Por último, en lo referente a las perspectivas de futuro, la mayoría esperan que mejoren o que continúen como están, concretando algunas inquietudes personales como el avance médico en lo que se refiere al "placer sexual» de las personas transexuales. En cuanto a la opinión de los/as entrevistados/as sobre cómo será la integración social de las personas transexuales dentro de 10 años, haciendo referencia a las nuevas generaciones, todos coinciden es que será o esperan que sea mucho mejor que en la actualidad y que en el pasado. Por tanto, se espera que la sensibilización social aumente y que la educación en la diversidad sexual sea una realidad.

En cuanto a los resultados de los cuestionarios, la pregunta sobre si existe un tratado desfavorable por cuestión de identidad sexual en Canarias (1 nada frecuente; 6 muy frecuente), devuelve un valor medio de 4,04. En las preguntas relacionadas con la figura del/la trabajador/a social, el $94 \%$ de las personas participantes considera que esta profesión debería formar parte de los equipos de atención a la transexualidad, atribuyendo a la misma una importancia alta, pues la media es de un 5,09 ( 1 nada importante; 6 muy importante). En cuanto a las áreas en las que resultaría más útil su desempeño profesional, destacan la prevención del bullying o acoso escolar $(72,61 \%)$, organización de campañas contra la discriminación social $(60,71 \%)$ e intervención en las problemáticas familiares (59,52\%).

Un 50\% de los participantes afirma que las personas transexuales forman un grupo de especial vulnerabilidad, siendo un $47 \%$ los que opinan que lo son en determinadas ocasiones. El apoyo familiar es uno de los pilares más importantes con los que puede contar una persona transexual. Así lo corroboran todos los participantes de las encuestas. Pese a ello, un 9,6\% no ha contado con el apoyo de su familia y un $20,5 \%$ lo ha tenido en ocasiones.

Para finalizar, de un total de 72 respuestas, un $54,2 \%$ sí se ha sentido discriminado/a o excluido/a por ser una persona transexual, un 59\%, en los últimos años y el 16\% en la última semana. Los espacios de ocio (piscinas, gimnasios, restaurantes) son los contextos en los que más se han percibido situaciones de discriminación. 


\section{DISCUSIÓN Y CONCLUSIONES}

Vistos los resultados y teniendo en cuenta los antecedentes, se pueden extraer las siguientes conclusiones de la investigación.

Como se puede observar en los datos obtenidos, tanto el CIS (2013) como las personas transexuales de este estudio coinciden en la alta percepción de discriminación hacia el colectivo en nuestro país.

Los resultados en el ámbito sanitario muestran una gran proporción de personas que detectan una la falta de formación y/o información del personal sanitario sobre la transexualidad, estando acorde con lo recogido en el estudio de Esteva $e t$ al. (2012), por lo que se puede entender que, varios años después de esta investigación, la situación sigue siendo insatisfactoria.

En relación con el ámbito familiar, muchas familias rechazan la realidad en un principio, pero la aceptan con el paso del tiempo, lo que coincide con lo hallado por Martín (2006). En la actualidad se dispone de más información y de más apoyo familiar, debido al cambio social que se está produciendo en las nuevas generaciones. De esta manera, se espera que, en un futuro, la integración social de las personas transexuales sea mucho mejor.

Todos los resultados de este estudio confirman que la familia es el pilar más importante en el que se puede apoyar una persona transexual, coincidiendo igualmente en la importancia de que el Trabajo Social intervenga en los aspectos relacionados con problemáticas familiares, tercer ítem en importancia.

En el ámbito educativo, el Eurobarómetro (2015) señala que el 71\% de europeos y un $83 \%$ de espańoles estarían dispuestos a permitir el material escolar con contenido sobre la identidad de género, coincidiendo con las personas transexuales entrevistadas, que lo ven como algo muy importante para evitar situaciones discriminatorias en el aula y en el resto de contextos. En cuanto al uso de los baños y vestuarios, las personas entrevistadas lo reconocen como un foco de conflicto, aun estando recogido en la Ley 8/2014 de no discriminación por motivos de identidad de género, que su uso debe estar basado en la identidad manifestada por el menor.

Por otra parte, en el ámbito laboral, el CIS (2013) afirma que el 24,9\% de espańoles considera que ser transexual afecta a la hora de conseguir un trabajo, motivo por el cual, y según lo recogido en las entrevistas, las personas transexuales deben o suelen ocultar esta realidad para lograr emplearse o evitar situaciones adversas. Además, esta dificultad de acceso, reafirmada en las entrevistadas, es tratada por Ley 8/2014 de no discriminación por motivos de identidad de género.

El contexto comunitario, aunque en menor medida, también se identifica como foco de conflictos: el CIS (2013) recoge que al 5,1\% de la población le molestaría tener como vecino a una persona transexual; esto se relaciona con el $44,1 \%$ de participantes en la encuesta que afirma que la comunidad es un entorno de especial vulnerabilidad (tercer ítem en importancia de esta cuestión).

Para finalizar, las entrevistas, los cuestionarios y el CIS (2013) coinciden, en términos generales, en la percepción que existe acerca de la insuficiencia de actuaciones por parte de las administraciones públicas para evitar la discriminación hacia personas transexuales. 
Sin duda, sería de interés ampliar la muestra participante y proseguir con estudios que analicen y profundicen con detalle algunos de los contextos mencionados, puesto que estos gozarían de gran valor a la hora de crear e implementar proyectos de intervención social. Todo ello, desde la perspectiva profesional del Trabajo Social para generar material científico que mejore la eficacia de la disciplina hacia el colectivo transexual.

Recibido: 2-3-2018; aceptado: 21-6-18 


\section{BIBLIOGRAFÍA}

Alder, A. (2013). Realidad Jurídica y Social del Derecho a la Orientación e Identidad de Género (tesis doctoral). Universidad de Salamanca, España.

Álvarez, L. (2008). «VIH/SIDA y transexualidad». Crítica, (953), pp. 55-57.

Armaza, E. y Atienza, E. (2014). «La transexualidad: aspectos jurídico-sanitarios en el ordenamiento español». Salud Colectiva, 10 (3), pp. 365-377.

Aventín, N. (2015). «El asociacionismo y el empoderamiento de las familias herramientas de aceptación de la situación de transexualidad en menores de edad. La transexualidad como parte de la diversidad». Endocrinología Pediátrica, 6 (2), pp. 52-57.

Centro de Investigaciones Sociológicas (2013). Percepción de la Discriminación en España: estudio $n .^{\circ}$ 3000. Disponible en https://goo.gl/Emd5wu.

Esteva, I., Gómez-Gil, E., Cruz, M., Martínez-Tudela, J., Bergero, T., Olveira, G. y Grupo Gidseen, (2012). «Organización de la asistencia a la transexualidad en el sistema sanitario público español». Gaceta Sanitaria, 26 (3), pp. 203-210.

European Commission (2015). Special Eurobarometer 437 "Discrimination in the EU in 2015». Disponible en https://goo.gl/EmYqoa.

Fernández, N. (2010). Necesidades, calidad de vida y salud psicológica de las personas transexuales (tesis doctoral). Universidad de Salamanca, España.

Herrero, I. y Díaz, C. (2009). La situación de las personas transgénero y transexuales en Euskadi. Disponible en https://goo.gl/PV4vd5.

Martín, D. (2006). La transexualidad, diversidad de una realidad. Disponible en http://www.madrid. org/bvirtual/BVCM007057.pdf.

Rubio, J. (2009). «Aspectos sociológicos de la transexualidad». Nómadas. Revista Crítica de Ciencias Sociales y Jurídicas, 21, pp. 361-380.

VIDAL, À. (2006). «El papel de la familia en el trastorno de identidad sexual», en E. Gómez e I. Esteva, Ser transexual. Dirigido al paciente, a su familia, y al entorno sanitario, judicial y social. Barcelona: Editorial Glosa, pp. 365-674.

\section{REFERENCIAS LEGISLATIVAS}

Convenio Europeo de Derechos Humanos. Tribunal Europeo de Derechos Humanos. Council of Europe F-67075 Strasbourg cedex: https://goo.gl/kcxmmc.

LEY 3/2007 de 22 de marzo, reguladora de la rectificación registral de la mención relativa al sexo de las personas. Publicado en $B O E$ núm. 65 de 16 de marzo de 2007.

LEY 8/2014, de 28 de octubre, de no discriminación por motivos de identidad de género y de reconocimiento de los derechos de las personas transexuales. Publicado en $B O E$ núm. 281, de 20 de noviembre de 2014, pp. 94850 a 94860 . Referencia: BOE-A-2014-11995.

Resolución del Parlamento Europeo, de 12 de septiembre de 1989, sobre la discriminación de los transexuales. DOC A 3-16/89: https://goo.gl/Mtkqba. 
